\title{
TLR4 Gene Polymorphisms and Susceptibility to Gastric Carcinoma and Epstein-Barr Virus-associated Gastric Carcinoma in Northern China
}

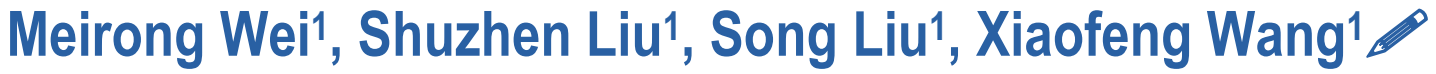

\begin{abstract}
${ }^{1}$ Departments and institutions from which the study originates: Department of Medical Microbiology, Qingdao University Medical College, Qingdao, China
\end{abstract}

\begin{abstract}
Background: Various polymorphisms in toll-like receptor (TLR) genes have been identified and are associated with susceptibility to various malignancies, such as gastric carcinoma (GC), hepatocellular carcinoma, papillary thyroid cancer, and prostate cancer. However, little is known about the polymorphisms of TLR genes and the susceptibility to GC in Northern China, especially to Epstein-Barr virus-associated GC (EBVaGC). The aim of our study was to evaluate the potential associations between the single nucleotide polymorphisms (SNPs) of TLR genes and the risk of GC, especially to EBVaGC. Methods: Polymorphisms of the TLR4 (rs10759932 and rs11536889) gene were assessed by polymerase chain reaction-restriction fragment length polymorphism (PCR-RFLP) in 43 cases of EBVaGC and 110 cases of EBV-negative GC (EBVnGC). 100 cases of peripheral blood samples from healthy individuals were also examined. Results: As for the TLR4 gene rs10759932, there was significant differences between the GC group and the control group in both genotype and allelic frequency. As for the TLR4 gene rs11536889, there was no association between the GC group and control. In all the indicators, no polymorphisms were found to be related to EBVaGC in the studied population. Conclusions: The polymorphisms of TLR4 gene rs10759932 were associated with susceptibility to GC in Shandong Province of China. There was no interaction between EBV and TLR gene polymorphisms in EBVaGC.
\end{abstract}

Keywords: Gastric Carcinoma, Epstein-Barr Virus-Associated Gastric Carcinoma, Toll-Like Receptor Gene Polymorphisms

\begin{abstract}
Introduction
Toll-like receptors (TLRs) are a kind of cell surface receptors and intracellular, mainly expressed in the surface of macrophages and dendritic cells, act as the first barrier of body defense to pathogen invasion. They are also the bridges that cross from the non-specific immunity to specific immunity. ${ }^{[1,2]}$ The study found that TLRs were germ-line-encoded innate immune sensors that distinguish conserved microbial structures and host alarmins and signal, then induced the expression of MHC proteins, costimulatory molecules, and inflammatory mediators by macrophages, neutrophils, dendritic cells, and other cell types. ${ }^{[3]}$ These processes activate immediate and early mechanisms of innate host defense, as well as initiate and elaborate adaptive immune responses. A total of 10 TLRs are expressed in humans. It is found that TLR expression is not only in immune cells, but also in a variety of cancer cells, and in inflammatory tumor cells which indicates it may play an important role in
\end{abstract}

tumorigenesis. Consequently, the activation of TLRs works in initiating the inflammatory response, especially plays an role in the inflammatory response of gastric mucosa. ${ }^{[4,5]}$ Recent studies have found that variations in TLR genes and imbalance of TLR signaling might lead to an unbalanced ratio between pro-and anti-inflammatory cytokines and thus contribute to a higher risk of developing chronic inflammatory diseases and cancers. ${ }^{[6-8]}$
Despite a main decline in incidence and mortality rates over several decades, gastric cancer (GC) still keeps a major cause of morbidity and mortality worldwide. ${ }^{[9]}$ According to global cancer statistics, 952,000 new cases were diagnosed in 2012, which occupied $6.8 \%$ of all cancers. ${ }^{[10]} \mathrm{GC}$, with high incidence in Eastern Europe, East Asia, and parts of Central and South America, is the fifth most common type of cancer and the third leading reason of cancer-related death worldwide. The incidence cases of GC in China 
account for $42 \%$ of the above worldwide estimation, with Chinese ethnicity determined as an independent risk factor for the development of GC in multiracial studies. ${ }^{[9,11,12]}$ It was found that the Helicobacter pylori (H. pylori) was the main etiological risk factor for GC, which progresses through a complex process, developing from gastritis, to gastric atrophy, intestinal metaplasia, dysplasia, and finally to carcinoma. ${ }^{[13]}$ However, colonization with $\mathrm{H}$. pylori can lead to various outcomes. Nearly all H. pylori positive subjects have chronic gastritis, but only $1 \%-2 \%$ develop to stomach cancer. ${ }^{[14]}$ Those information indicated that other factors were likely to be involved in gastric tumorigenesis, such as EBV and host genetic factors. The relationship between EBV infection and gastric cancer has also been confirmed. EBVaGC accounted for about $10 \%$ of the total number of gastric cancer. ${ }^{[15]}$ Although the ratio of EBVaGC is not very high, the large absolute number is due to the high morbidity and mortality of gastric cancer in the world.

Epstein-Barr virus (EBV), a herpes virus, is an important DNA tumor virus associated with a variety of tumors which is characterized by a capacity to establish lifelong latent infection both in B cell and epithelial cells. It has been proved that more than $95 \%$ of human beings worldwide are haunting infected with EBV. ${ }^{[16]}$ In order to remain the latent infection, EBV must resist the elimination of host defense mechanisms. There for, most individual infect EBV in early adulthood and primary infection is usually asymptomatic. It is still unclear that the exact mechanism of EBV in these associated malignant tumor although the gene products of EBV are well-known to induce cellular transformation. TLRs induced signaling in response to herpesvirus-unrelated infections lead to B-cell activation and their proliferation, finally the reactivation of EBV. ${ }^{[17,18]}$

To date, several studies have explored the role of innate immunity gene polymorphisms on gastric carcinogenesis. ${ }^{[19-21]}$ Polymorphism in the TLR4 gene had been shown to have an important role in some diseases. Studies have shown different results regarding the association between TLR4 polymorphisms and gastric cancer. ${ }^{[19,20,22]}$ And polymorphisms of the TLR4 gene have been reported to be connected with the risk of gastric cancer in a Brazilian population. ${ }^{[23]}$ However, little is known about its probable role in the natural history of EBV infections. Additionally, potent ethnic differences exist in the distribution of those polymorphisms. ${ }^{[5]}$
Consequently, TLR4 polymorphisms should be studied as possible susceptibility ingredients in the development of EBVaGC. Based on the information above, we hypothesized that some SNPs of TLR4 may be related to the occurrence of GC, especially to EBVaGC. The aims of the present work were to investigate whether polymorphisms of TLR4 (rs11536889 and rs10759932) contributed to the development of GC especially to EBVaGC.

\section{Materials and Methods \\ Ethics statement}

This study was approved by the Medical Ethics Committee of the Medical College of Qingdao University and was accomplished after written informed consent was acquired from all subjects. This study was implemented in accordance with the guidelines of the 1975 Declaration of Helsinki.

\section{Study population}

In this case-control study, EBV positivity in GC tissues was decided by in situ hybridization of EBV-encoded small RNA 1 (EBER1), as previously depicted. ${ }^{[24]}$ The case groups comprised 153 cases, including 43 cases of EBVaGC and 110 cases of EBVnGC. The control group was composed of 100 cases healthy subjects (50 men and 50 women), with a mean age of $55.6 \pm 1.3$ years (range 40-80 years), mainly blood donors. The EBVnGC group comprised 110 individuals (55 men and 55 women) with a histopathologically confirmed diagnosis of gastric cancer (Lauren's classification), with a mean age of $58.2 \pm 10.2$ years (range $42-87$ years). The EBVaGC group comprised 43 cases (23 men and 20women) with a mean age of $56.1 \pm 12.2$ years (range 38-79 years). All the control cases are healthy individuals, and the exclusion of various infections and autoimmune disease, especially to eliminate chronic stomach disease. The clinical and pathological features of two groups were matched. No significant differences were in age and gender with case groups, and all individuals were ethnically classified as Chinese Han.

\section{Tissue DNA extraction}

DNA was extracted from fresh tumor tissues and whole blood specimens by a standard method using proteinase $\mathrm{K}$ digestion and phenol-chloroform purification. A QIAamp DNA FFPE Tissue Kit (QIAGEN GmbH, Hilden, Hilden, Germany) was used to extract the DNA from paraffin-embedded tumor tissues. The extracted DNA was prepared for next polymerase chain reaction. 
TLR4 Gene Polymorphisms and Susceptibility to Gastric Carcinoma and Epstein-Barr Virus-associated Gastric Carcinoma in Northern China

Table 1 The primer sequences and PCR product size of TLR4.

\begin{tabular}{|c|c|c|}
\hline SNPs & Primers & Product size/bp \\
\hline \multirow[t]{2}{*}{ TLR4 rs10759932 } & F: 5'- TTTGTATAATTTGACTACCATTGCGT -3' & 139 \\
\hline & R: 5`- CATTTTTTCACATCTTCACCAGC -3’’ & \\
\hline \multirow[t]{4}{*}{ TLR4 rs11536889 } & F 1: 5‘-TTTGATGGACCTCTGAATCTC-3’' & $397 b p+184 b p(G G)$ \\
\hline & R1: 5‘- TTTTCTCAATGATAACATCCACTC-3’ & $397 b p+256 b p+184 b p(G C)$ \\
\hline & F2: 5‘- CTTGACCAC ATTTTGGGAAC-3’ & $397 b p+256 b p(C C)$ \\
\hline & R2:5‘-TTCCAATTTCTCTATATCCTTGATGA-3’ & \\
\hline
\end{tabular}

\section{Polymerase chain reaction amplification}

The polymerase chain reaction (PCR) technique was used to detect the polymorphisms in the TLR4 gene (rs10759932 and rs11536889). The primer sequences and the sizes of PCR products were shown in Table 1. PCR was performed with $2.0 \mu \mathrm{L}$ of DNA extracts (100 $\mathrm{ng} / \mu \mathrm{L})$ in a $25-\mu \mathrm{L}$ reaction mixture containing standard PCR buffer, $1.5 \mathrm{mM} \mathrm{MgCl} 2,200 \mu \mathrm{M}$ dNTP, $0.5 \mu \mathrm{M}$ of each primer, and 1.0 U Taq DNA polymerase. The PCR protocol was 5 minutes of initial denaturation at $94^{\circ} \mathrm{C}$, followed by 40 cycles of 1 minute at $94^{\circ} \mathrm{C}, 1$ minute at $55^{\circ} \mathrm{C}$, and 1 minute at $72^{\circ} \mathrm{C}$, then a 10 -minute final extension at $72^{\circ} \mathrm{C}$. The amplified DNA was visualized on a $2 \%$ agarose gel with ethidium bromide staining.

\section{Enzymatic digestion of PCR products}

PCR-RFLP technique was used to detect the Polymorphism of TLR4 (rs10759932 and rs11536889) in cancer and healthy control group. The amplified product of TLR4 (rs10759932) was then digested by Hha I (Fermentas International Inc, Canada) at $37^{\circ} \mathrm{C}$ for 15 minutes, then analyzed by vertical gel electrophoresis on a 12\% Polyacrylamide gel and visualized with ethidium bromide staining. The TLR4 (rs11536889) polymorphism was genotyped with PCR combined with confronting two-pair primers (PCR-CTPP) ${ }^{[25]}$. The amplified DNA was visualized on a $2 \%$ agarose gel with ethidium bromide staining.

To validate the PCR-RFLP and PCR-CTPP results, $10 \%$ of the samples were randomly selected to sequence.

\section{Statistical analyses}

The Chi-square test was used to compare the differences in each group regarding genotype and allele frequencies. Non-conditional logistic regression was used to compare the odds ratio (OR) and $\mathrm{P}$ values to indicate the relevance of each genotype and the risk of EBVaGC and EBVnGC. Significance was set at $\mathrm{P}<0.05$. Statistical analyses were conducted using SPSS 17.0 Statistical software (SPSS, Chicago, IL, USA).

\section{Results}

\section{Analysis of the TLR4 gene (rs10759932 and rs11536889) polymorphisms}

The length of the specifically amplified band of the TLR4 gene (rs10759932) was 139 bp. All 40 cases of EBVaGC, 110 cases of EBVnGC, and 100 cases of blood donors were positive for this band. After digestion with HhaI, the homozygous wild type genotype TT remained as only one $139 \mathrm{bp}$ band. The homozygous mutant genotype $\mathrm{CC}$ showed two bands, namely, $117 \mathrm{bp}$ and $22 \mathrm{bp}$. The heterozygous genotype CT showed three bands, 139 bp, 117 bp and 22 bp (Fig. 1). Analysis of the representative sequence was demonstrated in Figure 1. There was no significant difference between EBVaGC and EBVnGC. The differences in the distribution of genotype and allele frequency between the GC patients and the blood donors were statistically significant. The distributions of TLR4 (rs10759932) genotypes and alleles in patients and controls were listed in Tables 2 and 3 . 
TLR4 Gene Polymorphisms and Susceptibility to Gastric Carcinoma and Epstein-Barr Virus-associated Gastric Carcinoma in Northern China
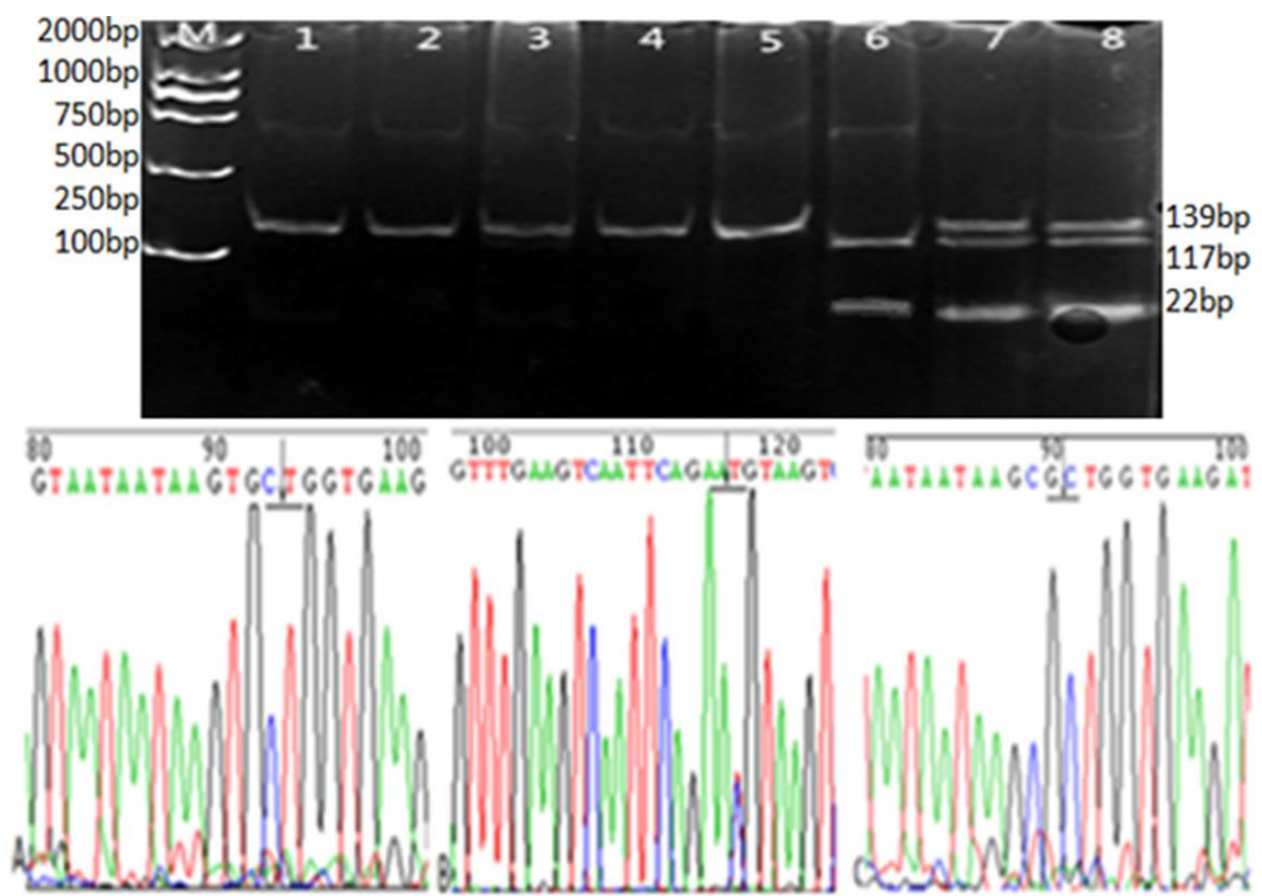

Figure 1: The results of RFLP analysis of TLR4 rs10759932 genotyping.

M: PCR marker DL2000; 1, 2, 3, 4, 5: TT Genotype; 7, 8: TC Genotype; 6: CC Genotype; A, representative samples (Type TT) Which lack of HhaI restriction site; $\mathrm{B}$, representative samples (Type TC) which possess HhaI site in the corresponding region; $\mathrm{C}$, representative samples (Type CC) which possess HhaI site. Arrow indicates the HhaI restriction site.

Determination of the TLR4 (rs11536889) genotype was based on the presence of the specific band. As indicated by the electrophoresis results, the amplified DNA was
$184 \mathrm{bp}$ for $\mathrm{G}$ allele, $256 \mathrm{bp}$ for $\mathrm{C}$ allele, and $397 \mathrm{bp}$ for common band. Representative gel for genotyping was shown in Figure 2. Analysis of the representative sequence was demonstrated in Figure 2. The associations between the EBVaGC and EBVnGC groups, the GC group, and the control group were not statistically significant in both genotype and allelic frequency. The distributions of TLR4 (rs11536889) genotypes and alleles in patients and controls were listed in Tables 2 and 3.
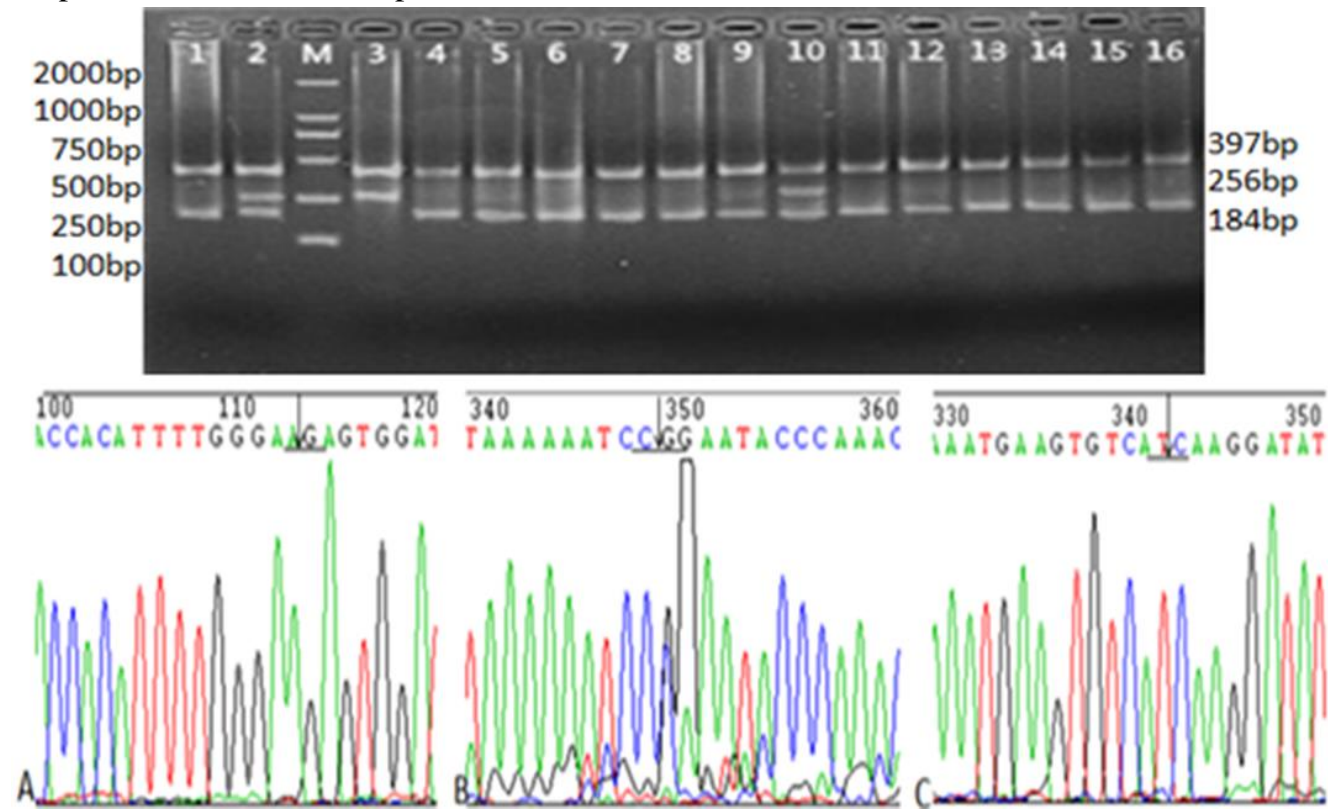

Figure 2: PCR analysis for TLR4 rs11536889 genotyping.

M: PCR marker DL2000; 1, 4, 5, 6, 7, 8, 9, 12, 13, 14, 15, 16: GG Genotype; 2, 10: GC Genotype; 3: CC Genotype; A, representative samples (Type GG); B, representative samples (Type GC); C, representative samples (Type CC). Arrow indicates the mutation site. 
TLR4 Gene Polymorphisms and Susceptibility to Gastric Carcinoma and Epstein-Barr Virus-associated Gastric Carcinoma in Northern China

\begin{tabular}{|c|c|c|c|c|c|}
\hline TLR4 SNP & Genotype/Allele & $\mathrm{GC}(\%)$ & Control (\%) & $\mathrm{P}$ & OR $(95 \% \mathrm{CI})$ \\
\hline \multirow[t]{12}{*}{ rs10759932(T/C) } & $\mathrm{TT}$ & $90(60.0)$ & $42(42.0)$ & & 1 \\
\hline & $\mathrm{TC}$ & $53(35.3)$ & $40(40.0)$ & 0.086 & $0.618(0.357-1.072)$ \\
\hline & $\mathrm{CC}$ & $7(4.7)$ & $18(18.0)$ & $<0.01$ & $0.181(0.070-0.468)$ \\
\hline & Total & 150 & 100 & & \\
\hline & $\mathrm{T}$ & $233(77.7)$ & $124(62.0)$ & & 1 \\
\hline & $\mathrm{C}$ & $67(22.3)$ & $76(38.0)$ & $<0.01$ & $0.469(0.316-0.696)$ \\
\hline & Recessive mode & & & & \\
\hline & Others & $143(95.3)$ & $82(82.0)$ & & 1 \\
\hline & $\mathrm{CC}$ & $7(4.7)$ & $18(18.0)$ & 0.001 & $0.223(0.089-0.556)$ \\
\hline & Dominant mode & & & & \\
\hline & TT & $90(60.0)$ & $42(42.0)$ & & 1 \\
\hline & Others & $60(40.0)$ & $58(58.0)$ & 0.005 & $0.483(0.289-0.807)$ \\
\hline \multirow[t]{12}{*}{ rs11536889(G/C) } & GG & $95(62.1)$ & $64(64.0)$ & & 1 \\
\hline & GC & $54(35.3)$ & $34(34.0)$ & 0.804 & $1.070(0.628-1.824)$ \\
\hline & $\mathrm{CC}$ & $4(2.6)$ & $2(2.0)$ & 1.000 & $1.347(0.240-7.575)$ \\
\hline & Total & 153 & 100 & & \\
\hline & $\mathrm{G}$ & $244(79.7)$ & $162(81.0)$ & & 1 \\
\hline & $\mathrm{C}$ & $62(20.3)$ & $38(19.0)$ & 0.728 & $1.083(0.691-1.699)$ \\
\hline & Recessive model & & & & \\
\hline & Others & $149(97.4)$ & $98(98.0)$ & & 1 \\
\hline & $\mathrm{CC}$ & $4(2.6)$ & $2(2.0)$ & 1.000 & $1.315(0.236-7.320)$ \\
\hline & Dominant model & & & & \\
\hline & GG & $95(62.1)$ & $64(64.0)$ & & 1 \\
\hline & Others & $58(37.9)$ & $36(36.0)$ & 0.759 & $1.085(0.637-1.831)$ \\
\hline
\end{tabular}


TLR4 Gene Polymorphisms and Susceptibility to Gastric Carcinoma and Epstein-Barr Virus-associated Gastric Carcinoma in Northern China

Table 3 The comparison of TLR4 gene polymorphism in EBVaGC and EBVnGC.

\begin{tabular}{|c|c|c|c|c|c|}
\hline TLR4 SNP & Genotype/Allele & $\operatorname{EBVaGC}(\%)$ & EBVnGC (\%) & $\mathrm{P}$ & OR $(95 \% \mathrm{CI})$ \\
\hline \multirow[t]{12}{*}{ rs10759932(T/C) } & TT & $19(47.5)$ & $71(64.5)$ & & 1 \\
\hline & $\mathrm{TC}$ & $18(45.0)$ & $35(31.8)$ & 0.090 & $1.922(0.898-4.114)$ \\
\hline & $\mathrm{CC}$ & $3(7.5)$ & $4(3.6)$ & 0.190 & $2.803(0.577-13.610)$ \\
\hline & Total & 40 & 110 & & \\
\hline & $\mathrm{T}$ & $56(70.0)$ & $177(80.5)$ & & 1 \\
\hline & $\mathrm{C}$ & $24(30.0)$ & $43(19.5)$ & 0.055 & $1.764(0.985-3.160)$ \\
\hline & Recessive model & & & & \\
\hline & Others & $37(92.5)$ & $106(96.4)$ & & 1 \\
\hline & $\mathrm{CC}$ & $3(7.5)$ & $4(3.6)$ & 0.384 & $2.149(0.459-10.053)$ \\
\hline & Dominant model & & & & \\
\hline & TT & $19(47.5)$ & $71(64.5)$ & & 1 \\
\hline & Others & $21(52.5)$ & $39(35.5)$ & 0.06 & $2.012(0.966-4.189)$ \\
\hline \multirow[t]{6}{*}{ rs11536889(G/C) } & GG & $23(53.5)$ & $72(65.5)$ & & 1 \\
\hline & $\mathrm{GC}$ & $18(41.9)$ & $36(32.7)$ & 0.231 & $1.565(0.750-3.265)$ \\
\hline & $\mathrm{CC}$ & $2(4.7)$ & $2(1.8)$ & 0.264 & $3.130(0.417-23.490)$ \\
\hline & Total & 43 & 110 & & \\
\hline & $\mathrm{G}$ & $64(74.4)$ & $180(81.8)$ & & 1 \\
\hline & $\mathrm{C}$ & $22(25.6)$ & $40(18.2)$ & 0.148 & $1.547(0.855-2.800)$ \\
\hline
\end{tabular}

Recessive model

$\begin{array}{ccccc}\text { Others } & \text { 41(95.3) } & \text { 108(98.2) } & & 1 \\ \text { CC } & 2(4.7) & 2(1.8) & 0.314 & 2.634(0.359-19.322)\end{array}$

Dominant model

\begin{tabular}{ccccc} 
GG & $23(53.5)$ & $72(65.5)$ & & 1 \\
Others & $20(46.5)$ & $38(34.5)$ & 0.170 & $1.648(0.805-3.373)$ \\
\hline
\end{tabular}




\section{Discussion}

TLRs have the ability to recognize extremely conserved structures particular to microbial pathogens-PAMPs (Pathogen-Associated Molecular Patterns). ${ }^{[26]}$ Stimulation of TLRs by PAMPs leads to the activation of intracellular signaling pathways which leads to the activations of nuclear factor NF-kB and secretion of cytokines. ${ }^{[17,18]}$ More and more studies found that the function of TLRs has supplied potential insight into the cancer development. TLR-dependent mechanisms benefit to activate effectors of innate immunity, thereby enlarge the adaptive immune response to cancer. Epidemiological and genetic studies advised that chronic inflammation might play a significant role in the development of several human cancers, such as GC. Recent study found that subjects with TLR4 and TLR2 polymorphisms had an increased risk of severe inflammation and following development of hypochlorhydria and gastric atrophy, which were regarded as the most significant precancerous abnormalities. ${ }^{[27]}$ In this case-control study of GC, we investigated the associations of TLR4 (rs10759932, rs11536889) with the risk of EBVnGC and EBVaGC in Chinese Han population of Shandong Province.

TLR4 is a significant lipo-polysaccharide (LPS) receptor in gastric epithelial cell signaling transduction and plays crucial roles in the development and progression of gastric cancer (GC). Growing evidence showed that polymorphisms of TLR4 had impacted on risk of cancer including gastric cancer. ${ }^{[19]}$ Numerous studies have investigated TLR4 polymorphisms in different populations and different diseases. Furthermore, polymorphisms of TLR4, such as Asp299Gly and Thr399Ile, can lead to persistent inflammation, advising the possibility of increased genetic susceptibility to cancers in subjects carrying TLR4 polymorphisms. ${ }^{[28,29]}$ The SNPs Asp299Gly and Thr399Ile of TLR4 occur with a frequency of 5\%-10\% in Caucasians, but are relatively rare in Asian populations. ${ }^{[30]}$ Liu et al. studied TLR4 Asp299Gly and Thr399Ile polymorphisms in Northern China with susceptibility to Gastric Carcinoma. ${ }^{[31]}$ The results showed that no SNPs were found in the two SNPs. Huang et al. found that rs10759932 genotypes (TC/CC) of the TLR4 gene were associated with a significantly decreased risk of gastric cancer in the high-risk population. ${ }^{[32]}$ Recently, researchers proved the biological significance of a genetic variation of the TLR4 gene named rs11536889. A large research including prostate cancer patients and age-matched controls from Sweden demonstrated an association between TLR4 (rs11536889) polymorphisms and prostate cancer. ${ }^{[33]}$ Hishida et al. observed that TLR4 (rs11536889) genotypes were related to severe gastric atrophy in helicobacter pylori-sero positive Japanese subjects. ${ }^{[34]}$ These observations advised that the SNP of TLR4 might affect human inflammatory and/or malignant diseases. However, Zhang et al. failed to reveal an association between TLR4 (rs11536889) and the risk of cancer after analyzing prostate, gastric and hepatocellular cancers as a group. ${ }^{[35]}$ Castano-Rodriguez found that polymorphisms of rs11536889 showed clear association with $H$. pylori-related GC in Chinese. ${ }^{[36]}$ Our results demonstrated that the TLR4 gene (rs10759932) polymorphisms were associated with susceptibility to GC in Shandong Province of Northern China. The genotype $\mathrm{CC}$ was a protective factor for gastric cancer. Our results were consistent with the results from Huang, ${ }^{[32]}$ and our data suggested that mutation of TLR4 gene (rs10759932) might play an important protective role in the development of GC. We also found that the polymorphism of TLR4 (rs11536889) had nothing to do with the risk of GC. These discrepancies could be due to limited sample sizes, analysis of different disease and ethnic populations, and multigenic effects. In addition, each individual gene was likely to contribute only a modest risk to GC.

Several host's factors may play an important role in promoting EBV reactivation and replication, such as immunodepression, inflammation or chronic immune stimulation. Stimulation of TLRs by microbial pathogen-associated molecular patterns and endogenous damage-associated molecular patterns leads to the immune system activation and may facilitate polyclonal proliferation of EBV-infected cells and/or EBV reactivation. ${ }^{[37]}$ The activation of TLR2, TLR3, TLR7, and TLR9 by EBV would avoid activating innate immune. ${ }^{[38]}$ Many studies have investigated the association between TLRs and EBV in various diseases. ${ }^{[39-41]}$ The interaction between TLR polymorphisms and EBVaGC was rarely discussed in former studies. In the present research, we focused on the likely being of such an interaction. However, we have found no evidence of an interaction between the polymorphism of TLR4(rs10759932 and rs11536889) and EBVaGC. More SNPs in the TLR genes are required further investigation to clarify the possible interaction.

However, our research has some limitations. First, inherent selection bias cannot be completely excluded. Second, the cancer cases and the controls are from one hospital and may not be representative of the entire target populations. In addition, the sample size of EBVaGC in our research was limited. The cause of gastric cancer is very complex. Many factors such as diet, race, genetic background, living environment and lifestyle, may lead to differences in cancer susceptibility. Genetic polymorphisms may interact with these factors to involvement with the risk of GC. Therefore, we need to detect the polymorphisms of TLR4 gene in other sites, and increase the patients with chronic inflammatory gastric diseases as the control group. More attention should be paid to the importance of environmental factors and host factors on the tumorgenesis of gastric cancer.

In summary, this study revealed that a significant difference existed between the GC group and healthy blood donors with regard to the presence of the TLR4 (rs10759932) gene polymorphisms, both in genotype and allele frequency in Northern China. Moreover, we found no interaction between EBV and TLR 
TLR4 Gene Polymorphisms and Susceptibility to Gastric Carcinoma and Epstein-Barr Virus-associated Gastric Carcinoma in Northern China

polymorphisms in EBVaGC in Shandong Province of Northern China.

\section{Acknowledgments}

The research was supported by the grants from: Specialized Research Fund for the Doctoral Program of Higher Education (20133706110001), Natural Science Foundation of Shandong Province (ZR2011CM016), Science and Technology of Qingdao City, China (13-1-3-50-jch).

\section{References}

1) Janssens S, Beyaert R. Role of Toll-like receptors in pathogen recognition. Clin Microbiol Rev 2003;16(4):637-646.

2) Akira S, Takeda K, Kaisho T. Toll-like receptors: critical proteins linking innate and acquired immunity. Nat Immunol 2001;2(8):675-680

3) Medvedev AE. Toll-like receptor polymorphisms, inflammatory and infectious diseases, allergies, and cancer. $\mathrm{J}$ Interferon Cytokine Res 2013;33(9):467-484.

4) Peek RM, Jr., Fiske C, Wilson KT. Role of innate immunity in Helicobacter pylori-induced gastric malignancy. Physiol Rev 2010;90(3):831-858.

5) Kutikhin AG. Impact of Toll-like receptor 4 polymorphisms on risk of cancer. Hum Immunol 2011;72(2):193-206.

6) Roses RE, Xu M, Koski GK, et al. Radiation therapy and Toll-like receptor signaling: implications for the treatment of cancer. Oncogene 2008;27(2):200-207.

7) Macagno A, Napolitani G, Lanzavecchia A, et al. combination and timing: the signal integration model of dendritic cell activation. Trends Immunol 2007;28(5):227-233.

8) $\mathrm{Xu} \mathrm{S}$, Koski GK, Faries M, et al. Rapid high efficiency sensitization of CD8+ $\mathrm{T}$ cells to tumor antigens by dendritic cells leads to enhanced functional avidity and direct tumor recognition through an IL-12-dependent mechanism. J Immunol 2003;171(5):2251-2261.

9) Lochhead P, El-Omar EM. Gastric cancer. Br Med Bull 2008;85:87-100.

10) Ferlay J, Soerjomataram I, Dikshit R, et al. Cancer incidence and mortality worldwide: sources, methods and major patterns in GLOBOCAN 2012. Int J Cancer 2015;136(5):E359-386.

11) Brenner $H$, Rothenbacher $D$, Arndt V. Epidemiology of stomach cancer. Methods Mol Biol 2009;472:467-477.

12) Goh KL, Cheah PL, Md N, et al. pylori as risk factors for gastric cancer in Malaysia: A prospective case control study. Am J Gastroenterol 2007;102(1):40-45.

13) Correa P. A human model of gastric carcinogenesis. Cancer Res 1988;48(13):3554-3560.

14) Scholte GH, van Doorn LJ, Cats A, et al. Genotyping of Helicobacter pylori in paraffin-embedded gastric biopsy specimens: relation to histological parameters and effects on therapy. Am J Gastroenterol 2002;97(7):1687-1695.

15) Fukayama M, Chong JM, Kaizaki Y. Epstein-Barr virus and gastric carcinoma. Gastric Cancer 1998;1(2):104-114.

16) Luzuriaga K, Sullivan JL. Infectious mononucleosis. N Engl J Med 2010;362(21):1993-2000.

17) Finberg RW, Kurt-Jones EA. Viruses and Toll-like receptors Microbes Infect 2004;6(15):1356-1360.

18) Hopkins PA, Sriskandan S. Mammalian Toll-like receptors: to immunity and beyond. Clin Exp Immunol 2005;140(3):395-407.

19) Hold GL, Rabkin CS, Chow WH, et al. A functional polymorphism of toll-like receptor 4 gene increases risk of gastric carcinoma and its precursors. Gastroenterology 2007;132(3):905-912.

20) Garza-Gonzalez E, Bosques-Padilla FJ, Mendoza-Ibarra SI, et al. Assessment of the toll-like receptor 4 Asp299Gly, Thr399Ile and interleukin-8 -251 polymorphisms in the risk for the development of distal gastric cancer. BMC Cancer 2007;7:70.

21) Tahara T, Arisawa T, Wang F, et al. Toll-like receptor $2-196$ to 174del polymorphism influences the susceptibility of Japanese people to gastric cancer. Cancer Sci 2007;98(11):1790-1794.

22) Wu MS, Cheng TY, Shun CT, et al. Functional polymorphisms of CD14 and toll-like receptor 4 in Taiwanese Chinese with Helicobacter pylori-related gastric malignancies. Hepatogastroenterology 2006;53(71):807-810.

23) de Oliveira JG, Silva AE. Polymorphisms of the TLR2 and TLR4 genes are associated with risk of gastric cancer in a Brazilian population. World J Gastroenterol 2012;18(11):1235-1242.

24) Parkin DM, Bray F, Ferlay J, et al. Global cancer statistics, 2002. Ca-a Cancer Journal for Clinicians 2005;55(2):74-108.

25) Hamajima N, Saito $T$, Matsuo $K$, et al. Polymerase chain reaction with confronting two-pair primers for polymorphism genotyping. Jpn J Cancer Res 2000;91(9):865-868.

26) Pasare $C$, Medzhitov R. Toll-like receptors: linking innate and adaptive immunity. Microbes Infect 2004;6(15):1382-1387.

27) El-Omar EM, Ng MT, Hold GL. Polymorphisms in Toll-like receptor genes and risk of cancer. Oncogene 2008;27(2):244-252.

28) Smith MF, Jr., Mitchell A, et al. Toll-like receptor (TLR) 2 and TLR5, but not TLR4, are required for Helicobacter pylori-induced NF-kappa B activation and chemokine expression by epithelial cells. Journal of Biological Chemistry 2003;278(35):32552-32560.

29) Kato I, Canzian F, Plummer M, et al. Polymorphisms in genes related to bacterial lipopolysaccharide/peptidoglycan signaling and gastric precancerous lesions in a population at high risk for gastric cancer. Dig Dis Sci 2007;52(1):254-261.

30) Perez-Piqueras J, Jaqueti J, Navarro-Gallar F, et al. [Prevalence of serum markers of hepatitis B virus, in health personnel at a general hospital]. Rev Esp Enferm Apar Dig 1989;76(6 Pt 2):609-611.

31) Liu S, Wang $X$, Shi $Y$, et al. Toll-like receptor gene polymorphisms and susceptibility to Epstein-Barr virus-associated and -negative gastric carcinoma in Northern China. Saudi J Gastroenterol 2015;21(2):95-103.

32) Huang $\mathrm{H}, \mathrm{Wu} \mathrm{J}$, Jin $\mathrm{G}$, et al. A 5 '-flanking region polymorphism in toll-like receptor 4 is associated with gastric cancer in a Chinese population. J Biomed Res 2010;24(2):100-106.

33) Zhou L, Wei B, Xing C, et al. Polymorphism in 3'-untranslated region of toll-like receptor 4 gene is associated with protection from hepatitis B virus recurrence after liver transplantation. Transpl Infect Dis 2011;13(3):250-258.

34) Hishida A, Matsuo K, Goto Y, et al. Toll-like receptor $4+3725$ G/C polymorphism, Helicobacter pylori seropositivity, and the risk of gastric atrophy and gastric cancer in Japanese. Helicobacter 2009;14(1):47-53.

35) Zhang $\mathrm{K}$, Zhou $\mathrm{B}$, Wang $\mathrm{Y}$, et al. The TLR4 gene polymorphisms and susceptibility to cancer: a systematic review and meta-analysis. Eur J Cancer 2013;49(4):946-954.

36) Castano-Rodriguez N, Kaakoush NO, Pardo AL, et al. Genetic polymorphisms in the Toll-like receptor signalling pathway in Helicobacter pylori infection and related gastric cancer. Hum Immunol 2014;75(8):808-815.

37) Petrara MR, Freguja R, Gianesin K, et al. Epstein-Barr 
TLR4 Gene Polymorphisms and Susceptibility to Gastric Carcinoma and Epstein-Barr Virus-associated Gastric Carcinoma in Northern China

virus-driven lymphomagenesis in the context of human immunodeficiency virus type 1 infection. Front Microbiol 2013;4:311.

38) van Gent M, Braem SG, de Jong A, et al. Epstein-Barr virus large tegument protein BPLF1 contributes to innate immune evasion through interference with toll-like receptor signaling. PLoS Pathog 2014;10(2):e1003960.

39) Moumad K, Lascorz J, Bevier M, et al. Genetic polymorphisms in host innate immune sensor genes and the risk of nasopharyngeal carcinoma in North Africa. G3 (Bethesda) 2013;3(6):971-977.

40) He JF, Jia WH, Fan Q, et al. Genetic polymorphisms of TLR3 are associated with Nasopharyngeal carcinoma risk in Cantonese population. BMC Cancer 2007;7:194.

41) Valente RM, Ehlers E, Xu D, et al. Toll-like receptor 7 stimulates the expression of Epstein-Barr virus latent membrane protein 1. PLoS One 2012;7(8):e43317. 